\title{
Histone deacetylase inhibitors in Hodgkin lymphoma
}

\author{
Daniela Buglio • Anas Younes
}

Received: 27 October 2010 /Accepted: 28 October 2010 /Published online: 3 December 2010

(C) The Author(s) 2010. This article is published with open access at Springerlink.com

\begin{abstract}
Summary Although Hodgkin lymphoma (HL) is considered one of the most curable human cancers, the treatment of patients with relapsed and refractory disease, especially those who relapse after autologous stem cell transplantation, remains challenging. Furthermore, because of the young age of these patients, the impact of early mortality on the number of years lost from productive life is remarkable. Patients with relapsed HL post stem cell transplantation currently have no curative therapy, and are in need for new drugs and novel treatment strategies. While no new drugs have been approved for the treatment of patients with HL in more than three decades, several new agents are demonstrating promising results in early clinical trials. This review will focus on the emerging role of histone deacetylase inhibitors in patients with relapsed HL.
\end{abstract}

Keywords Hodgkin lymphoma Role of histone deacetylase inhibitors

\section{Introduction}

Epigenetics is a heritable process that alters gene expression without changing the DNA sequence [1]. This process includes DNA and chromatin modifications by methylation, acetylation, phosphorylation, ubiquitylation, and sumolyation. Epigenetic processes are natural and essential to many organism functions, but if they occur improperly, they may result in major adverse health effects. The best known epigenetic process is DNA methylation, which involves the

D. Buglio $\cdot$ A. Younes $(\bowtie)$

Department of Lymphoma/Myeloma,

1515 Holcombe Boulevard, Box \# 429, Houston, TX 77030, USA

e-mail: ayounes@mdanderson.org addition of a methyl group $\left(\mathrm{CH}_{3}\right)$, which primarily occurs at the $5^{\prime}$ position of the pyrimidine ring of the cytosine. The resulting methylcytosine is mainly found in cytosine-guanine $(\mathrm{CpG})$ islands [2]. The presence of 5-methylcytosine in the promoter of specific genes alters the binding of transcriptional factors and other proteins to DNA and recruits methylDNA-binding proteins and histone deacetylases that compact the chromatin around the gene-transcription start site. Both mechanisms block transcription and cause gene silencing.

Another significant epigenetic process is the posttranscriptional modification of histones. Chromatin is the complex of histone proteins and DNA that is tightly bundled to fit into the nucleus. The complex can be modified by histone acetylation, which alters chromatin structure to influence gene expression [3]. In general, tightly folded chromatin tends to be shut down, or not expressed, while more open chromatin is functional, or expressed. Among all the epigenetic research conducted so far, the most extensively studied disease family is cancer, and the evidence linking epigenetic processes with cancer continues to grow $[1,4,5]$.

\section{Histone deacetylases (HDACs)}

Post-transcriptional histone modification plays an important role in regulating gene transcription, and is mediated by a variety of enzymes, including histone acetyltransferases (HATs) and histone deacetylation (HDACs) [3]. These enzymes mediate acetylation and deacetylation of specific lysine amino acid residues on histone tails. In general, histone $\mathrm{H} 3$ and $\mathrm{H} 4$ acetylation is associated with open chromatin status favoring gene transcription, whereas histone deacetylation is associated with closed chromatin status favoring transcription repression and gene silencing. The balance between HATs and HDACs is critical for 
regulating the expression of a variety of genes that are involved in cell proliferation, survival, angiogenesis, and immunity [6-8]. To date, 18 HDACs have been identified in humans $[9,10]$. HDACs are grouped in two major categories: zinc-dependent HDACs and NAD-dependent HDACs. Furthermore, HDACs are classified into four major classes: Class I includes HDAC 1, 2, 3, 8, and 11; Class II includes HDAC 4, 5, 6, 7, 9, and 10; Class III includes homologues of yeast SIRT 1-7, and Class IV, which currently includes only HDAC 11 (Fig. 1). Class III is NAD-dependent, whereas classes I, II, and IV are zinc dependent. In addition to histones, HATs mediate posttranslational protein acetylation on lysine residues in a variety of nonhistone proteins, including transcription factors (p53, STAT3, MYC, GATA-1, GATA-2, E2F, NF$\kappa B$, nuclear receptors, HIF- $1 \alpha$, and TEL), $\alpha$-tubulin, and heat shock protein-90 (HSP90) [3, 11-13].

\section{HDAC inhibitors (HDACis)}

Because HDACs target histones and a variety of nonhistone proteins that control cell survival, proliferation, angiogenesis, and immunity, they became attractive targets for cancer therapy. At the present time, clinical grade pharmacologic inhibitors of the zinc-dependent HDACs are available for clinical trials, and two inhibitors, vorinostat and romidepsin, have already been approved by the FDA for the treatment of patients with relapsed cutaneous T-cell

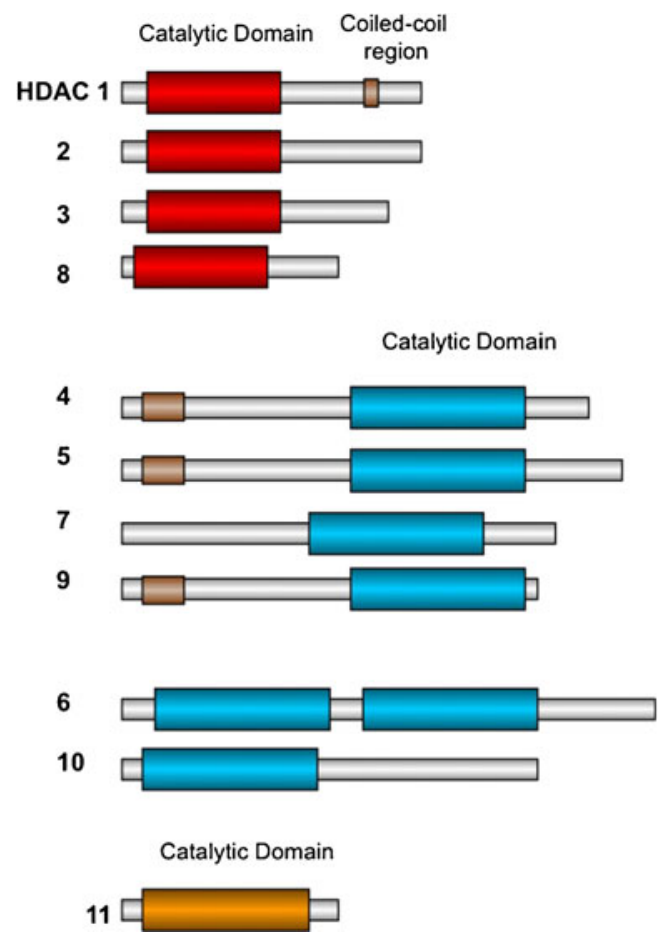

Fig. 1 Zinc-dependent HDACs lymphoma $[14,15]$. Vorinostat inhibits a wide range of HDACs, including classes I and II, and therefore is frequently referred to as pan-DAC inhibitor. Although Romidepsin is considered a pan-DAC inhibitor as well, it shows stronger activity against class I than against the class II HDACs [9]. In contrast, entinostat (SNDX-275) and mocetinostat (MGCD0103) are selective inhibitors that preferentially inhibit class I HDACs and to a less extent class IV (Fig. 2) [16].

HDACis mediate antitumor activity by modulating a variety of survival pathways $[3,9,13,17]$. HDACis induce cell cycle arrest by regulating the expression of p21 and p27 cell-cycle proteins, in addition to inducing cell death by activating the intrinsic and extrinsic apoptosis pathways. In fact, a variety of HDACi have been shown to transcriptionally regulate several components of the death pathways, including upregulation of proapoptotic proteins (Bak, Bax, Apaf-1, Bad, Bim, Bid, caspase3, and 9), and repression of antiapoptotic proteins (Bcl2, Bcl-xL, Mcl-1, XIAP, cFLIP). Furthermore, HDACi have been reported to upregulate the transcription of TRAIL and its death receptors and Fas ligand (C95L) and Fas [18-20].

Several HDACis are currently being evaluated for the treatment of cancer, and there is accumulating evidence that this treatment strategy is effective for the treatment of hematologic malignancies in general, and lymphoid malignancies in particular [13, 19, 21, 22]. In fact, several HDACis have recently reported promising clinical activity, including clinical remissions, in patients with Hodgkin lymphoma (HL) and non-Hodgkin lymphoma (NHL) [23-29].



Fig. 2 A schematic grouping of HDAC inhibitors and their mechanisms of action 


\section{Rationale for using epigenetic-based therapy in $\mathrm{HL}$}

HRS cells are B cells with epigentically-silenced B-cell genes Molecular analysis demonstrated that the malignant Hodgkin and Reed-Sternberg (HRS) cells of Hodgkin lymphoma (HL) are of B-cell origin. However, HRS cells infrequently express B-cell antigens. This loss of B-cell phenotype has been reported to be epigenetically regulated and is thought to enable HRS cells to evade immunosurveillance. For example, gene expression profiles of HRS cells revealed decreased mRNA levels for nearly all B-cell lineage-specific genes [30]. The decrease in mRNA expression was associated with downregulation of protein expression in primary HL cases, as determined by immunohistochemistry, including CD20, CD19, Syk, Lck, Blk, Oct-2, and B-cell receptor [30]. Furthermore, microdissected primary HRS cells and HL cell lines are downregulated in HL. Importantly, the expression of these silenced genes could be re-induced by the DNA hypomethylating agent 5 -aza-deoxycytidine [31, 32]. A variety of HDAC inhibitors have demonstrated promising pre-clinical activity in HL cell lines by inducing cell cycle arrest and apoptosis [28]. AT the molecular level, HDACis upregulated $\mathrm{p} 21$, activated the caspase pathway, downregulated STAT6 expression, and altered cytokine and chemokine secretion [33-35]. In fact, we have recently demonstrated that vorinostat decreased the expression and secretion of $\mathrm{T}_{\mathrm{h}}$ 2-type cytokines and chemokines, including Thymus and Activation-Regulated Chemokine (TARC/CCL17) and interleukin (IL)-5, and increased $\mathrm{T}_{\mathrm{h}} 1$-type cytokines/chemokines, including a profound increase in IP-10 levels, suggesting that HDACi may alter the microenvironment surrounding HRS cells to favor cell death [35].

Epigenetic therapy induces the expression of Epstein-Barr virus antigens and tumor-associated antigens in HRS cells, which are potential targets for cytotoxic $T$ cells EpsteinBarr virus (EBV)-associated antigens are known to be expressed by a proportion of HRS cells, however, only the subdominant EBV antigens LMP1 and LMP2 are expressed and provide T-cell target antigens. EBV-specific T cells that are enriched for LMP-specific T-cell clones have been expanded from patients and administered to patients with relapsed disease [36, 37]. Unfortunately, $\mathrm{T}$ cells specific for the viral latent nuclear antigens, EBNAs $3 \mathrm{~A}, 3 \mathrm{~B}$ and $3 \mathrm{C}$ and the immediate early lytic cycle antigen, BZLF1, BRLF1 and BMLF1 dominate the human immune response to EBV. If these proteins could be reactivated in EBVpositive HRS cells, then their susceptibility to EBV-specific T-cell lines would be increased. Both HDACis and hypomethylating agents induce EBV gene expression. HDACs reactivate EBV from latency to replication, so that the immunogenic lytic cycle antigens are expressed [38-
40], while azacytidine can switch latently infected cells from a minimal form of latency (LMP1,LMP2 and EBNA1) to an unrestricted latency in which all EBNAs (2, 3's and-LP) and LMPs are expressed. Thus, these drugs may transform poorly antigenic tumors into highly immunogenic tumors and increase the number of available target antigens for T-cell recognition immunodominant viral proteins. In addition, combining different agents like chemotherapy and HDAC inhibitors results in synergistic effects on activating lytic viral replication [40]. Since only about $20 \%$ of patients with relapsed HL have EBV-positive tumors, it is also important to determine if other tumorassociated, T-cell target antigens are upregulated by epigenetic therapy. Cancer Testis Antigens (CTAs) are known to comprise at least 44 families of genes or isoforms. Among these, 19 families are purely testisrestricted, while others are expressed in one or more somatic tissues. Some CTAs are expressed in lymphoma [41, 42], and provide potential T-cell targets. CTA-specific $\mathrm{T}$ cells from melanoma patients have demonstrated therapeutic benefits, including complete remissions. CTAspecific CTL could have similar beneficial effects in EBV-negative lymphomas. Recently, hypomethylating agents have been shown to induce the expression of several CTAs in cultured tumor cell lines (including lymphomas) [43]. Such upregulated expression has been reported for several MAGE members - LAGE-1, SSX-2, CAGE, and NY-ESO-1. Demethylation proved necessary and sufficient for MAGE-A1 gene expression in melanoma cell lines, suggesting that methylation is a primary mechanism of transcription control. Histone deacetylase (HDAC) inhibitors, on their own, or in combination with hypomethylating agents, can also induce CTA expression, including MAGE, SSX, and NY-ESO-1 family members [43]. Recent data suggested that HDACi may upregulate the expression of CTA in HRS, suggesting HDACis may enhance the activity of EBV-directed cellular therapy in HL [33]. Since epigenetically enhanced CTA expression may activate endogenous CTA-specific $\mathrm{T}$ cells in vivo, this may induce epitope spreading and facilitate the ex vivo activation and expansion of these normally low frequency CTA-specific T cells.

\section{Clinical results with HDACi in patients with HL}

A variety of HDACis are currently being evaluated in patients with relapsed HL (Table 1). The following is a brief update on the current status of HDACis in patients with relapsed HL. Interestingly, the expression pattern of HDAC enzymes varied among different types of lymphoma [44]. Consistent with their ubiquitous distribution in normal 
Table 1 Summary results of HDACi in patients with relapsed cHL

\begin{tabular}{lllllllc}
\hline Drug & Target & Route & Phase & Number of evaluable patients & PR & CR & PR + CR (ORR) \\
\hline MGCD0103 [26] & HDACs & Oral & II & 21 & 6 & 2 & $8(38 \%)$ \\
ITF2357 [49] & HDACs & Oral & II & 13 & 0 & 0 & 0 \\
Panobinostat [51] & HDACs & Oral & I & 20 & 8 & 0 & $8(40 \%)$ \\
Panobinostat [48] & HDACs & Oral & II & 53 & 10 & 1 & $11(21 \%)$ \\
Vorinostat [24] & HDACs & Oral & II & 25 & 1 & 0 & $1(4 \%)$ \\
\hline
\end{tabular}

tissues, class I enzymes were highly expressed in all Hodgkin and non-Hodgkin lymphoma cell lines and primary tumors studied, including the non-malignant reactive cells in $\mathrm{HL}$ microenvironment. In contrast, the class II enzyme HDAC6 was infrequently expressed, compared with HDACs 5, 8, and 10. These results suggest that HDAC6 may not be an important therapeutic target in selected lymphoid malignancies [44].

Vorinostat (SAHA) Vorinostat, which inhibits HDAC classes I and II, has a potent antiproliferative activity in HL cell lines, as it induces cell cycle arrest and apoptosis, in addition to synergizing with chemotherapy [45]. Furthemore, Vorinostat inhibited STAT6 phosphorylation and transcription in HL cell lines, an effect that was associated with decreased expression and secretion of Th2-type cytokines and chemokines (TARC and IL-5). This effect was associated with and increases of in Th1-type cytokines/ chemokines (IP-10) [45]. The in vivo activity of vorinostat was recently evaluated in a phase II study that was conducted by the Southwest Oncology Group (SWOG) in patients with relapsed HL [16]. Twenty-five patients were treated with $200 \mathrm{mg}$ vorinostat given orally twice per day for 14 days every 21-day cycle. Vorinostat produced modest clinical activity, as only 1 patient (4\%) achieved a partial remission.

Mocetinostat (MGCD-0103) Mecetinostat is a novel oral HDAC inhibitor that selectively inhibits HDAC 1, 2, 3 (class I) and 11 (class IV) isoforms, with no effect on classII HDACs [46]. The safety and efficacy of MGCD-0103 given orally 3 times per week ( 85 to $110 \mathrm{mg}$ starting doses) has been evaluated in a phase II study in patients with relapsed and refractory HL [26]. Patients were allowed to continue therapy up to 1 year in absence of heavy toxicity or disease progression. Of the 20 patients who were treated with 110-mg dose level, 7 (35\%) patients achieved partial or complete remissions. However, this dose level was poorly tolerated resulting in dose interruptions and reductions, and discontinuation of therapy after a median of 4.5 months. Subsequently, the study was revised to allow a lower starting dose of $85 \mathrm{mg}$ at the same schedule. Three of the $10(30 \%)$ patients enrolled on the reduced dose achieved partial remissions. Furthermore, grade 3 and 4 toxicity (mainly fatigue, with no significant hematologic toxicity) was reduced to $20 \%$. Overall, $80 \%$ of the 30 evaluable patients had some decrease in their tumor measurements. Although none of the patients developed significant EKG abnormalities, two patients developed significant pericardial effusions requiring discontinuation of therapy [47]. Importantly, Serum TARC levels were determined in 15 patients before and 1 week after initiation of therapy [26]. After 1 week of therapy serum TARC levels were decreased by at least $40 \%$ in five patients, and all achieved major clinical responses. Collectively, this data indicate that class I HDAC inhibitors have a potential therapeutic value in patients with HL.

Panobinostat (LBH 589) Panobinostat is one of the most potent HDACis in HL in vitro, with an IC50 in the nanomolar range [28]. Recently panobinostat was evaluated in a phase I trial in patients with hematologic malignancies that also included patients with relapsed HL [18]. Five of $13(38 \%)$ patients achieved partial remissions. The most common side effects were fatigue, thrombocytopenia, nausea, and diarrhea. Based on this promising clinical activity, a large international phase II study of panobinostat in relapsed HL, with an early report demonstrating a promising clinical activity in a large multicenter settings [48]. In a most recent update of the study, 61 patients have been enrolled and treated: median age 31 years (range 1870 ), and $48 \%$ had stage III/IV at initial diagnosis. Patients were heavily pretreated with a median number of prior regimens of 4 (range 2-6 regimens). Of 61 patients, 53 have completed two cycles of therapy and have had one post baseline imaging studies to evaluate response to theapy. Sixty-nine percent had tumor reduction, including one complete response and 10 partial responses The most common grade $3 / 4$ adverse events were thrombocytopenia (77\%), anemia (20\%), and neutropenia (16\%). Thrombocytopenia was reversible within an average of 1 week of dose interruption. No cases of grade 3/4 QTc prolongation have been reported. Thus, panobinostat has a promising clinical activity with a good safety profile. Based on this promising data, panobinostat-based combination studies are currently being conducted, including a phase I/II trial of 
panobinostat plus everolimus in patients with relapsed HL and non-Hodgkin lymphoma.

Entinostat (SNDX-275) Entinostat is an oral, class I isoform selective HDAC inhibitor. Phase 1 studies in leukemia demonstrated the agent has a long half-life and that weekly or every other week dosing is sufficient for antitumor activity. In vitro experiments demonstrated a potent activity of entinostat in HL-derived cell lines, with an IC50 of $0.4 \mu \mathrm{M}$ [33]. At the molecular level, entinostat induced its antiproliferative effect in HL cell lines by multiple mechanisms: it increased $\mathrm{H} 3$ acetylation, up-regulated p21 protein expression, and activated the intrinsic apoptosis pathway by down-regulating the anti-apoptotic X-linked inhibitor or apoptosis (XIAP) protein, which was associated with activation of caspase 9 and 3 [33]. Furthermore, entinostat had synergistic effects when combined with gemcitabine and bortezomib. Moreover, entinostat increased IL12 p40-70, IP10, and RANTES, and decreased the level of IL13 and IL4, in HL cell lines supernatants, thus favoring Th1-type cytokines/chemokines. Finally, entinostat induced the expression of a variety of tumor-associated antigens, including SSX2 and MAGE-A [33]. Based on this encouraging in vitro data, the safety and efficacy of entinostat is currently being evaluated in a phase 2 multi-center study in patient with relapsed or refractory HL. In this study entinostat is given $10 \mathrm{mg}$ (two $5 \mathrm{mg}$ tablets) orally, once every two weeks (days 1 and 15) in a 28 day cycle increasing to $15 \mathrm{mg}$ in absence of treatment related toxicity. Results of this study are expected to be available in 2010.

ITF 2357 ITF 2357 is a selective class I and class II HDAC inhibitor. The efficacy, safety, and tolerability of daily 100 $\mathrm{mg}$ doses of ITF 2357 in relapsed/refractory HL were investigated in a phase II clinical trial at the National Tumors Institute of Milan [49]. The preliminary results were presented at the 2008 ASCO meeting: 15 patients were enrolled and 13 were evaluable for response. Seven patients $(54 \%)$ had a stable disease, with a reduction in FDG-PET uptake in six patients (46\%) lasting at least 3 months; six patients had disease progression. Interestingly, a correlation was found between a decrease in serum TARC levels and the response to treatment in this study. On the basis of the single-agent activity of ITF 2357 and the documented synergistic activity of ITF 2357 and mechlorethamine in HL cell lines, a phase II trial of ITF 2357 combined with mechlorethamine was conducted by the same group [50]. Nineteen patients who unsuccessfully underwent prior autologous/allogeneic stem cell transplantation were enrolled. Preliminary data from 17 evaluable patients demonstrated that two patients $(12 \%)$ achieved complete and three (18\%) partial remissions. As expected, the major toxicity was hematologic, with seven patients experiencing grade III/IV neutropenia and eight having thrombocytopenia; four patients experienced infections during treatment. Taken together, these data suggest that ITF 2357 has encouraging clinical activity in relapsed/refractory HL.

\section{Conclusions}

Class I and pan-DAC inhibitors are promising new agents for the treatment of patients with relapsed HL. Future studies should examine predictive biomarkers, such as TARC levels and the pattern of HDAC enzyme expression in HL patients treated with HDACis to determine whether a certain pattern of expression may predict response to therapy. Furthermore, because HDAC inhibitors can modulate a variety of survival proteins, combination regimens with HDAC inhibitors should be investigated. Ultimately, these studies will hopefully improve our treatment strategies for patients with relapsed and refractory HL.

Conflict of interest Daniela Buglio: No conflict of interests Anas Younes: No conflict of interests

Logistical support during submission of this article was provided by Springer Healthcare LLC. This support was funded by Novartis.

Open Access This article is distributed under the terms of the Creative Commons Attribution Noncommercial License which permits any noncommercial use, distribution, and reproduction in any medium, provided the original author(s) and source are credited.

\section{References}

1. Jones PA, Baylin SB (2007) The epigenomics of cancer. Cell 128:683-692

2. Issa JP (2007) DNA methylation as a therapeutic target in cancer. Clin Cancer Res 13:1634-1637

3. Glozak MA, Seto E (2007) Histone deacetylases and cancer. Oncogene 26:5420-5432

4. Baylin SB (2005) DNA methylation and gene silencing in cancer. Nat Clin Pract Oncol 2(Suppl 1):S4-S11

5. Marsoni S, Damia G, Camboni G (2008) A work in progress: the clinical development of histone deacetylase inhibitors. Epigenetics 3:164-171

6. Heider U, Kaiser M, Sterz J et al (2006) Histone deacetylase inhibitors reduce VEGF production and induce growth suppression and apoptosis in human mantle cell lymphoma. Eur $\mathrm{J}$ Haematol 76:42-50

7. Wang S, Yan-Neale Y, Cai R, Alimov I, Cohen D (2006) Activation of mitochondrial pathway is crucial for tumor selective induction of apoptosis by LAQ824. Cell Cycle 5:1662-1668

8. Brogdon JL, Xu Y, Szabo SJ et al (2007) Histone deacetylase activities are required for innate immune cell control of Th1 but not Th2 effector cell function. Blood 109:1123-1130

9. Bolden JE, Peart MJ, Johnstone RW (2006) Anticancer activities of histone deacetylase inhibitors. Nat Rev Drug Discov 5:769-784

10. Minucci S, Pelicci PG (2006) Histone deacetylase inhibitors and the promise of epigenetic (and more) treatments for cancer. Nat Rev Cancer 6:38-51 
11. Glozak MA, Sengupta N, Zhang X, Seto E (2005) Acetylation and deacetylation of non-histone proteins. Gene 363:15-23

12. Schrump DS (2009) Cytotoxicity mediated by histone deacetylase inhibitors in cancer cells: mechanisms and potential clinical implications. Clin Cancer Res 15:3947-3957

13. Stimson L, Wood V, Khan O, Fotheringham S, La Thangue NB (2009) HDAC inhibitor-based therapies and haematological malignancy. Ann Oncol 20:1293-1302

14. Duvic M, Talpur R, Ni X et al (2007) Phase 2 trial of oral vorinostat (suberoylanilide hydroxamic acid, SAHA) for refractory cutaneous T-cell lymphoma (CTCL). Blood 109:31-39

15. Piekarz RL, Frye R, Turner $M$ et al (2009) Phase II multiinstitutional trial of the histone deacetylase inhibitor romidepsin as monotherapy for patients with cutaneous T-cell lymphoma. J Clin Oncol 27:5410-5417

16. Khan N, Jeffers M, Kumar S et al (2008) Determination of the class and isoform selectivity of small-molecule histone deacetylase inhibitors. Biochem J 409:581-589

17. Bi G, Jiang G (2006) The molecular mechanism of HDAC inhibitors in anticancer effects. Cell Mol Immunol 3:285-290

18. Beumer JH, Tawbi H (2010) Role of histone deacetylases and their inhibitors in cancer biology and treatment. Curr Clin Pharmacol 5(3):196-208

19. Lane AA, Chabner BA (2009) Histone deacetylase inhibitors in cancer therapy. J Clin Oncol 27:5459-5468

20. Borbone E, Berlingieri MT, De Bellis F et al (2010) Histone deacetylase inhibitors induce thyroid cancer-specific apoptosis through proteasome-dependent inhibition of TRAIL degradation. Oncogene 29:105-116

21. Prince HM, Bishton MJ, Harrison SJ (2009) Clinical studies of histone deacetylase inhibitors. Clin Cancer Res 15:3958-3969

22. Marks PA, Xu WS (2009) Histone deacetylase inhibitors: potential in cancer therapy. J Cell Biochem 107:600-608

23. O'Connor OA, Heaney ML, Schwartz L et al (2006) Clinical experience with intravenous and oral formulations of the novel histone deacetylase inhibitor suberoylanilide hydroxamic acid in patients with advanced hematologic malignancies. J Clin Oncol 24:166-173

24. Kirschbaum MH, Goldman BH, Zain JM et al (2007) Vorinostat (suberoylanilide hydroxamic acid) in relapsed or refractory hodgkin lymphoma: SWOG 0517. Blood (ASH Annual Meeting Abstracts) 110:2574

25. Kirschbaum MH, Zain JM, Popplewell L et al (2007) A phase 2 study of vorinostat (suberoylanilide hydroxamic acid, SAHA) in relapsed or refractory indolent Non Hodgkin Lymphoma. A California Cancer Consortium Study. Blood (ASH Annual Meeting Abstracts) 110:2568

26. Younes A, Pro B, Fanale M et al (2007) Isotype-selective HDAC inhibitor MGCD0103 decreases serum TARC concentrations and produces clinical responses in heavily pretreated patients with relapsed classical Hodgkin Lymphoma (HL). Blood (ASH Annual Meeting Abstracts) 110:2566

27. Younes A, Wedgwood A, McLaughlin P et al (2007) Treatment of relapsed or refractory lymphoma with the oral isotypeselective histone deacetylase inhibitor MGCD0103: interim results from a phase II study. Blood (ASH Annual Meeting Abstracts) 110:2571

28. Younes A (2009) Novel treatment strategies for patients with relapsed classical Hodgkin lymphoma. Hematology 2009:507519

29. Dickinson M, Ritchie D, DeAngelo DJ et al (2009) Preliminary evidence of disease response to the pan deacetylase inhibitor panobinostat (LBH589) in refractory Hodgkin Lymphoma. Br J Haematol 147:97-101

30. Schwering I, Brauninger A, Klein U et al (2003) Loss of the Blineage-specific gene expression program in Hodgkin and ReedSternberg cells of Hodgkin lymphoma. Blood 101:1505-1512
31. Ushmorov A, Ritz O, Hummel M et al (2004) Epigenetic silencing of the immunoglobulin heavy-chain gene in classical Hodgkin lymphoma-derived cell lines contributes to the loss of immunoglobulin expression. Blood 104:3326-3334

32. Ushmorov A, Leithauser F, Sakk O et al (2006) Epigenetic processes play a major role in B-cell-specific gene silencing in classical Hodgkin lymphoma. Blood 107:2493-2500

33. Khaskhely NM, Buglio D, Shafer J, Bollard CM, Younes A (2009) The Histone Deacetylase (HDAC) Inhibitor Entinostat (SNDX-275) targets Hodgkin Lymphoma through a dual mechanism of immune modulation and apoptosis induction. Blood (ASH Annual Meeting Abstracts) 114:1562

34. Buglio D, Mamidipudi V, Khaskhely NM et al (2009) The histone deacetylase inhibitor MGCD0103 down regulates CD30, activates $\mathrm{NF}-\mathrm{Kb}$, and synergizes with proteasome inhibitors by HDAC6 independent mechanism in Hodgkin Lymphoma. Blood (ASH Annual Meeting Abstracts) 114:3735

35. Buglio D, Georgiakis GV, Hanabuchi S et al (2008) Vorinostat inhibits STAT6-mediated TH2 cytokine and TARC production and induces cell death in Hodgkin lymphoma cell lines. Blood 112:1424-1433

36. Bollard CM, Aguilar L, Straathof KC et al (2004) Cytotoxic T lymphocyte therapy for Epstein-Barr virus+ Hodgkin's disease. J Exp Med 200:1623-1633

37. Bollard CM, Gottschalk S, Leen AM et al (2007) Complete responses of relapsed lymphoma following genetic modification of tumor-antigen presenting cells and T-lymphocyte transfer. Blood 110:2838-2845

38. Israel BF, Kenney SC (2003) Virally targeted therapies for EBVassociated malignancies. Oncogene 22:5122-5130

39. Feng WH, Hong G, Delecluse HJ, Kenney SC (2004) Lytic induction therapy for Epstein-Barr virus-positive B-cell lymphomas. J Virol 78:1893-1902

40. Feng WH, Kenney SC (2006) Valproic acid enhances the efficacy of chemotherapy in EBV-positive tumors by increasing lytic viral gene expression. Cancer Res 66:8762-8769

41. Chambost H, Van Baren N, Brasseur F et al (2000) Expression of gene MAGE-A4 in Reed-Sternberg cells. Blood 95:3530-3533

42. Colleoni GW, Capodieci P, Tickoo S, Cossman J, Filippa DA, Ladanyi M (2002) Expression of SSX genes in the neoplastic cells of Hodgkin's lymphoma. Hum Pathol 33:496-502

43. Shichijo S, Yamada A, Sagawa K et al (1996) Induction of MAGE genes in lymphoid cells by the demethylating agent 5-aza-2'deoxycytidine. Jpn J Cancer Res 87:751-756

44. Gloghini A, Buglio D, Khaskhely NM et al (2009) Expression of histone deacetylases in lymphoma: implication for the development of selective inhibitors. Br J Haematol 147:515-525

45. Buglio D, Georgakis GV, Hanabuchi S et al (2008) Vorinostat inhibits STAT6-mediated TH2 cytokine and TARC production and induces cell death in Hodgkin lymphoma cell lines. Blood 112:1424-1433

46. Fournel M, Bonfils C, Hou Y et al (2008) MGCD0103, a novel isotype-selective histone deacetylase inhibitor, has broad spectrum antitumor activity in vitro and in vivo. Mol Cancer Ther 7:759768

47. Martell RE, Garcia-Manero G, Younes A et al (2009) Clinical development of MGCD0103, an isotype-selective HDAC inhibitor: pericarditis/pericardial effusion in the context of overall safety and efficacy. Blood (ASH Annual Meeting Abstracts) 114:4756

48. Younes A, Ong T-C, Ribrag V et al (2009) Efficacy of panobinostat in phase II study in patients with relapsed/refractory Hodgkin Lymphoma (HL) after high-dose chemotherapy with autologous stem cell transplant. Blood (ASH Annual Meeting Abstracts) 114:923

49. Viviani S, Bonfante V, Fasola C, Valagussa P, Gianni AM (2008) Phase II study of the histone-deacetylase inhibitor ITF2357 in 
relapsed/refractory Hodgkin's lymphoma patients. J Clin Oncol 26:2008 (May suppl; abstract 8532)

50. Carlo-Stella C, Guidetti A, Viviani S et al (2008) Phase II trial of combination of the histone deacetylase inhibitor ITF2357 and meclorethamine demonstrates clinical activity and safety in heavily pretreated patients with relapsed/refractory Hodgkin
Lymphoma (HL). Blood (ASH Annual Meeting Abstracts) 112:2586

51. Spencer A, DeAngelo DJ, Prince HM et al (2008) Oral panobinostat (LBH589), a novel deacetylase inhibitor (DACi) demonstrates clinical activity in relapsed/refractory Hodgkin lymphoma (HL). Ann Oncol 19:136, Abstract 